University of Warwick institutional repository: http://go.warwick.ac.uk/wrap This paper is made available online in accordance with publisher policies. Please scroll down to view the document itself. Please refer to the repository record for this item and our policy information available from the repository home page for further information.

To see the final version of this paper please visit the publisher's website. Access to the published version may require a subscription.

Author(s): V. I. Khizhny, O. A. Mironov, E. H. C. Parker, P. J. Phillips, T. E. Whall, and M. J. Kearney

Article Title: Direct evidence for a piezoelectriclike effect in coherently strained SiGe/Si heterostructures

Year of publication: 1996

Link to published version: http://dx.doi.org/10.1063/1.117095

Publisher statement: none 


\title{
Direct evidence for a piezoelectriclike effect in coherently strained SiGe/Si heterostructures
}

\author{
V. I. Khizhny and O. A. Mironov \\ Institute of Radiophysics and Electronics, Ukrainian National Academy of Sciences, 12, Acad. Proscura St., \\ Kharkov 310085, Ukraine \\ E. H. C. Parker, ${ }^{\text {a) }}$ P. J. Phillips, and T. E. Whall \\ Department of Physics, University of Warwick, Coventry CV4 7AL, United Kingdom \\ M. J. Kearney \\ Department of Electronic and Electrical Engineering, Loughborough University of Technology, \\ Loughborough, Leics. LE11 3TU, United Kingdom
}

(Received 29 February 1996; accepted for publication 4 June 1996)

\begin{abstract}
A hybrid acoustic spectroscopy technique has been used to demonstrate the (reversible) conversion of high frequency electric fields into longitudinal acoustic waves within a modulation-doped pseudomorphic $\mathrm{Si} / \mathrm{Si}_{0.88} \mathrm{Ge}_{0.12} / \mathrm{Si}$ heterostructure. This provides compelling evidence for the existence of a piezoelectriclike coupling within such structures. (C) 1996 American Institute of Physics. [S0003-6951(96)03133-6]
\end{abstract}

Transmission electron microscopy ${ }^{1}$ and four circle $\mathrm{x}$-ray diffraction ${ }^{2}$ measurements have provided evidence for partial ordering of the $\mathrm{Si}$ and $\mathrm{Ge}$ atoms in the $\langle 111\rangle$ direction in strained SiGe layers grown on (001) Si substrates. The combination of strain and ordering is expected to lead to a piezoelectriclike effect in such systems, ${ }^{3,4}$ which is of interest since neither bulk silicon or germanium are piezoelectric. ${ }^{5}$ Indirect evidence from Raman scattering ${ }^{6,7}$ and hot-carrier Shubnikov-de Haas ${ }^{3,7}$ measurements supports the notion of a finite piezoelectric coupling, but the issue is controversial, and clearly a more direct experimental probe of possible piezoelectricity is desirable.

In the present work, a very sensitive hybrid acoustic spectroscopy technique ${ }^{8}$ has been used to successfully demonstrate the conversion of a high frequency (HF) electric field $E$ into a longitudinal acoustic wave within a $\mathrm{SiGe} / \mathrm{Si}$ strained layer sample. We have also established the converse effect and thus the reversibility of the process. The extent to which one can ascribe this novel observation to piezoelectric coupling is discussed below.

The experimental arrangement and measuring cell are shown in Figs. 1 and 2, respectively. The experiment was carried out using the standard resonant "pulse-on passage" technique ${ }^{8}$ [Fig. 2(a)], in which the sample was placed in a HF electric field parallel to the growth axis (z-axis) and acoustically coupled to one end of a $3.283 \mathrm{~cm}$ sapphire buffer rod. The (pulsed) field $E$ was created by an electrode in a coaxial cavity and was frequency tunable over the range 215-235 MHz; typically each pulse was of $1 \mu$ s duration

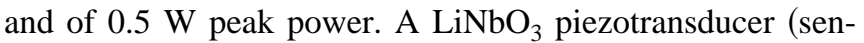
sitive to longitudinal waves only) was coupled to the other end of the buffer rod to register any longitudinal acoustic pulses created in the sample and then propagating along its $z$ axis. Acoustic contact between the sample and the buffer rod and the piezotransducer and the buffer rod was ensured by thin films of silicon hydrophobic fluid which solidified at $150 \mathrm{~K}$, enabling experiments to be carried out below this temperature. The results presented below were obtained at 77 $\mathrm{K}$. Given that the longitudinal velocity of sound in sapphire at $77 \mathrm{~K}$ is $\sim 1.12 \times 10^{6} \mathrm{~cm} \mathrm{~s}^{-1},{ }^{9}$ the expected delay for the first sound pulse would be $\sim 3 \mu$ s, with subsequent, weaker pulses due to multiple reflections along the rod being detected every $6 \mu$ s or so thereafter. The present setup is reminiscent of previous experiments carried out with diffusion layer ${ }^{10,11}$ and depletion layer transducers, ${ }^{11}$ but in our case the metal electrode on the surface of the sample has been replaced by one near to (but not in intimate contact with) the actual sample surface.

A typical sample studied (see, e.g., Ref. 12) is depicted in Fig. 2(b), and comprises a $p$-type modulation doped $\mathrm{Si}: \mathrm{B} / i-\mathrm{Si} / \mathrm{Si}_{0.88} \mathrm{Ge}_{0.12}$ heterostructure grown on an $n$-type $\mathrm{Si}$ substrate by solid source molecular beam epitaxy. This sample had a $4.2 \mathrm{~K}$ sheet carrier density of 1.8 $\times 10^{11} \mathrm{~cm}^{-2}$ and a $4.2 \mathrm{~K}$ mobility of 10800 $\mathrm{cm}^{2} \mathrm{~V}^{-1} \mathrm{~s}^{-1}$, and when subjected to a pulsed electric field of frequency $f=226 \mathrm{MHz}$ applied parallel to the $z$ axis (growth axis), the oscilloscope trace depicted in Fig. 3 was obtained. Ignoring the excitation pulse at $t \sim 0$, clear evidence is seen for an initial pulse [(b) in Fig. 3] detected by the transducer (with a delay corresponding to time-of-flight down the buffer rod) followed by subsequent echoes [(c) and (d) in Fig. 3]. The measured time-of-flight is slightly longer than the above estimate of $3 \mu \mathrm{s}$, but is consistent with estimate which also allow for the effect of the finite thickness $d=0.575 \mathrm{~mm}$ of the sample, which is dominated by the

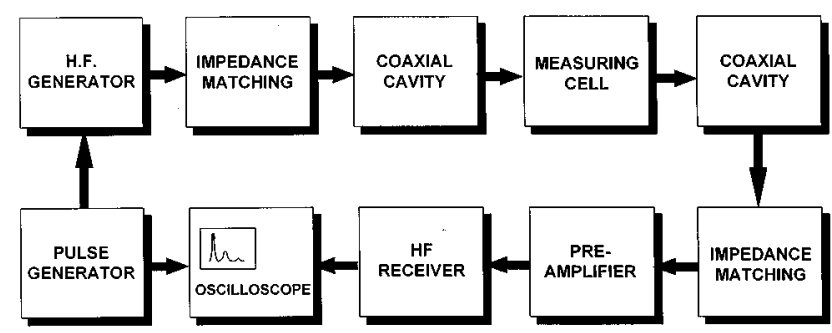

FIG. 1. Block diagram of the HF acoustic spectroscopy apparatus.

${ }^{\text {a)} E l e c t r o n i c ~ m a i l: ~ s e c 2 @ ~ @ s p e c . w a r w i c k . a c . u k ~}$ 


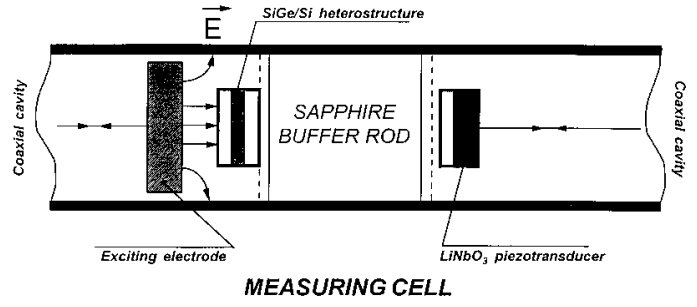

(a)

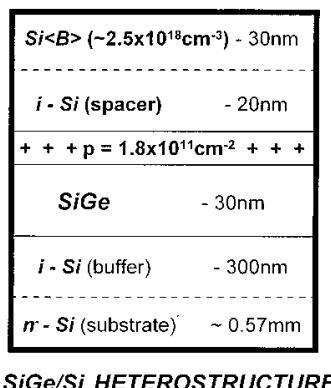

(b)

FIG. 2. (a) Measurement configuration, (b) schematic of the $\mathrm{Si}_{0.88} \mathrm{Ge}_{0.12} / \mathrm{Si}$ heterostructure.

thickness of the Si substrate. At $f=226 \mathrm{MHz}$ the amplitude of the initial detected signal $V_{2}$ was $110 \mathrm{~dB}$ below the electromagnetic input $V_{1}$ and its signal-to-noise ratio was 5-8 dB. Similar behavior was found with every other sample studied of the this same basic type, for a range of HF power levels and temperatures.

The results presented in Fig. 3 are deliberately nonoptimized with regard to the frequency, so as to illustrate the sensitivity of the present technique. On sweeping the frequency of the HF generator within the bandwidth of the $\mathrm{LiNbO}_{3}$ transducer $(\sim 15 \mathrm{MHz})$ and measuring the amplitude of the first detected pulse, the resonant nature of the acoustic response of the system is demonstrated (Fig. 4). Besides the principal maximum at $f=225.2 \mathrm{MHz}$ (where the amplitude of the initial detected signal $V_{2}$ was $\sim 90 \mathrm{~dB}$ below the electromagnetic input $V_{1}$ ), subsidiary (weaker) maxima at 218 $\mathrm{MHz}$ and $\sim 232.5 \mathrm{MHz}$ are also resolved. There is a natural interpretation of this in terms of Fabry-Perot-type interfer-

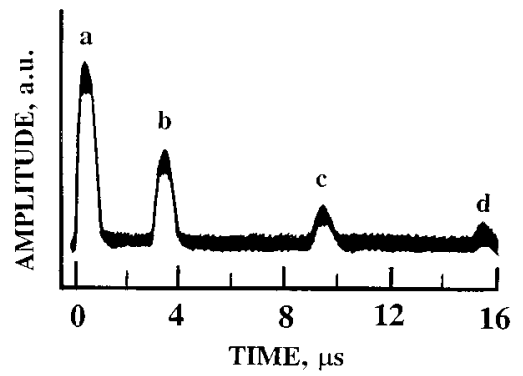

FIG. 3. Oscilloscope trace demonstrating excitation of sound at $77 \mathrm{~K}$ by a $\mathrm{HF}$ electric field of frequency $226 \mathrm{MHz}$. The pulse (a) at $t \sim 0$ corresponds to the electrical excitation pulse, the second pulse (b) is produced by the $\mathrm{LiNbO}_{3}$ transducer and occurs at a time closely corresponding to one transit of a longitudinal sound wave down the sapphire delay line. The first (c) and second (d) echoes are also shown.

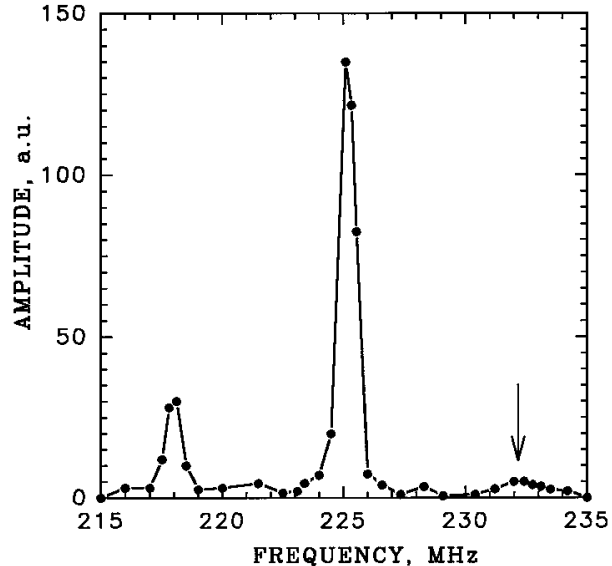

FIG. 4. Frequency response of the HF acoustic spectroscopy apparatus, illustrating Fabry-Perot resonances in the $\mathrm{SiGe} / \mathrm{Si}$ heterostructure.

ence occurring within the SiGe/Si sample itself. ${ }^{11}$ Taking into account the fact that the longitudinal velocity of sound $v_{l}$ in $(001) \mathrm{Si}$ at $77 \mathrm{~K}$ is $8.45 \times 10^{5} \mathrm{~cm} \mathrm{~s}^{-1}$, the experimentally observed fringe separation of $\sim 7.2 \mathrm{MHz}$ is in good agreement with the theoretical value $\left(\delta f=v_{l} / 2 d\right)$ of 7.3 MHz. The subsidiary maxima are weaker because they have been "convolved" with the response function of the $\mathrm{LiNbO}_{3}$ transducer itself. As the time-of-flight of the acoustic wave through the sample is much less than the duration of the HF pulse, we can expect to have a quasicontinuous acoustic wave within the sample. The acoustic resonance at 225.2 MHz has a quality factor $Q$ of about 300 .

We stress that the effect observed is reversible, in that not only could acoustic waves generated by the $E$ field in the sample be detected at the piezotransducer, but it was also possible to detect $E$ fields generated in the sample (by acoustic waves from the piezotransducer) at the cavity. Upon replacing the samples with several Si:B $\delta$-doped homojunction samples no effect was seen nor was any effect seen with a standard Si substrate. In other words, it only proved possible to generate the effect with the modulation-doped heterostructures. We comment further on these observations below.

Our favored interpretation of these results is to associate a finite piezoelectric stress constant $e(z)$ (for simplicity we do not use indices for $e_{i j}(z)$ tensor here) with the thin SiGe layer [within the Si layers and $\mathrm{Si}$ substrate $e(z)$ is assumed to be zero]. In response to an applied electric-field $E(z, t)$ $=E(z) e^{-i \omega t}$, the stress in the medium is given by

$$
\sigma(z, t)=c \varepsilon(z, t)-e(z) E(z, t),
$$

where $c$ is the elastic stiffness constant and $\varepsilon(z, t)$ is the strain. Since $\varepsilon(z, t)=\partial u(z, t) / \partial z$, the displacement $u(z, t)$ is given by ${ }^{11}$

$$
\frac{\partial^{2} u}{\partial z^{2}}-\frac{1}{v_{l}^{2}} \frac{\partial^{2} u}{\partial t^{2}}=\frac{\partial}{\partial z}\left(\frac{e(z) E(z, t)}{c}\right) .
$$

The right-hand side acts as a source term for the wave equation. Since the spatial derivative of $E(z)$ will be weaker than $e(z)$, the sources are predominantly localized at the $\mathrm{Si} / \mathrm{SiGe}$ interfaces. This suggests that one does not require a great thickness of SiGe to observe the effect. The intensity of ultrasound generated will be proportional to the cross-sectional 
area of the interface, ${ }^{11}$ and to maximize the effect one of the two interfaces should dominate (to avoid excessive cancellation). The build-in electric fields due to depletion and the heterostructure should favor the upper interface, much as in a conventional depletion layer transducer ${ }^{11}$ where ultrasound is generated piezoelectrically in regions where the built-in field intensity is greatest (where most of the external field is then dropped). A fuller analysis would take into account the fact that the source term will cause the sample to resonate, but this does not alter the essential physics of the situation.

According to the above model, our experiments are probing mainly local conditions at or near the interface. Near the interface, the symmetry of the piezoelectric tensor must be distinct from that of, say, GaAs (cubic, $43 \mathrm{~m}$ ), for which no effect would be seen in the geometry indicated. However, this is not unexpected. We suggest that the observed ordering ${ }^{1,2}$ at or near the interface is indicative of rhombohedral $(3 \mathrm{~m})$ symmetry, whereupon the nonvanishing component $e_{33}$ of the resulting piezoelectric tensor would lead to the effect. Of course, the theoretical situation is unclear, and the issue of symmetry and the strength of the indicated coupling must await further theoretical clarification. It is interesting to note, however, that cracks in bulk $\mathrm{Si}$, leading to a loss of inversion symmetry by a different mechanism, have been reported to lead to the generation of piezovoltages, ${ }^{13}$ showing the importance of treating local conditions correctly. It has even been suggested that strain without ordering might be sufficient to generate a piezoelectric coupling. ${ }^{4}$

One must acknowledge that there are alternative ways of generating ultrasound that have nothing to do with piezoelectricity. Such mechanisms are better known in the context of metals. ${ }^{14,15}$ The most studied case (see, e.g., Ref. 14 and references therein) concerns a transverse electromagnetic wave $(E \perp z)$ impinging on the surface of a metal. Electrons within the skin-depth $\delta$ of the surface respond to the $E$ field and can transfer momentum to the lattice via deformation potential coupling. The ions of the background lattice also respond to the $E$ field, but in the regime of the anomalous skin effect $(\delta<\ell$, where $\ell$ is the mean-free path of the carriers) the two forces can be locally unbalanced leading to generation of transverse acoustic waves at the surface. ${ }^{14}$ The geometry relevant to our experiment $(E \| z)$ has received much less attention, but it has been established that a similar mechanism can apply, leading this time to the generation of longitudinal sound waves. ${ }^{15}$ In addition to $\ell$ and $\lambda$, a relevant length scale is now the Debye (screening) length $L_{D}$ $=V_{F} / \omega_{p}$ (where $V_{F}$ is the Fermi velocity and $\omega_{p}$ is the plasma frequency); typically in metals $L_{D} \ll \lambda$ and the effect in enhanced, the larger the ratio $\ell / \lambda$. Could a similar mechanism explain the behavior we observe? An obvious difficulty is to explain why electroacoustic conversion should be absent for the Si:B delta-doped samples (unless somehow the lower mobility of these samples compared to the heterostructure is responsible). Direct quantitative analysis on the basis of the existing theory for bulk metals is unfortunately not feasible (the limited thickness of the hole gas and the fact that the carriers are only able to respond effectively in the plane of the hole gas are crucial complicating factors), and more theoretical work is needed before one can be certain that such a mechanism is even possible in the present struc- tures. On the other hand, it would perhaps to be unwise to rule out such mechanisms at this stage, and certainly unwise to rule out a possible mediation of any piezoelectric coupling by mobile carrier in the active samples.

Although experimentally awkward, preliminary attempts have been made to apply the $E$-field transverse to the $z$ axis of our samples, using a detecting transducer sensitive to shear waves. For piezoelectric coupling with rhombohedral symmetry one might expect shear wave generation in this configuration, but no effect was observed (nor was any effect observed with the $\delta$-layer samples or with any of the other samples discussed above). However, this could be due to many factors, such as in inefficient coupling of the $E$ field, a low absolute value of the $e_{15}$ component of the piezoelectric tensor, or nonoptimal detection. Further experimental work is needed to clarify the situation in this case.

In conclusion, we have provided the first direct experimental evidence for the (reversible) conversion of a high frequency electric field into a longitudinal acoustic wave within a $\mathrm{SiGe} / \mathrm{Si}$ strained layer sample. We believe our results provide strong evidence for a piezoelectriclike coupling within such structures, although further work (both theoretical and experimental) is clearly warranted. Our results also demonstrate the effectiveness of hybrid HF acoustic spectroscopy for investigating such phenomena as those solids in general.

Thanks are due to Professor V. I. Litvinov for communications about SiGe symmetry, and also to Professor M. Oszwaldowski, Dr. V. W. Rampton, and Dr. A. G. Kozorezov for fruitful discussions. This work is partially supported by an INTAS-93-1403 Grant, and by the Ukrainian Science and Technology Council (Project "KREMNII94"').

${ }^{1}$ A. Ourmazd and J. C. Bean, Phys. Rev. Lett. 55, 765 (1985).

${ }^{2}$ J. Z. Tischler, J. D. Budai, D. E. Jesson, G. Eres, P. Zschack, J.-M. Baribeau, and D. C. Houghton, Phys. Rev. B 51, 10947 (1995).

${ }^{3}$ Y. H. Xie, R. People, J. C. Bean, and K. W. Wecht, Appl. Phys. Lett. 49, 283 (1986).

${ }^{4}$ Y. H. Xie, R. People, J. C. Bean, and K. W. Wecht, J. Vac. Sci. Technol. B 5, 744 (1987).

${ }^{5}$ J. C. Phillips and J. A. Van Vechten, Phys. Rev. Lett. 22, 705 (1969).

${ }^{6}$ D. H. Lockwood, K. Rajan, E. W. Fenton, J.-M. Baribeau, M. W. Denhoff, Solid State Commun. 61, 465 (1987).

${ }^{7}$ O. A. Mironov, V. I. Khizhny, G. Braithwaite, E. H. C. Parker, P. J. Phillips, T. E. Whall, and V. P. Gnezdilov, Abstracts of 6th International Conference on Si-MBE, Council of Europe/European Parliament, Strasbourg (France), L-14, E-MRS Spring Meeting, 22nd-26th May, 1995.

${ }^{8}$ V. I. Khizhnyi, E. M. Ganapol'skyi, and A. P. Korolyuk, Physica C 185, 1377 (1991); V. I. Khizhnyi, E. M. Ganapol'skyi, and A. P. Korolyuk, Abstracts of 4th International Conference M2S HTSC IV Grenoble (France), p. 187 (WD-OPS 380), 5th-9th July, 1994.

${ }^{9}$ J. deKlerk, Phys. Rev. A 139, 1635 (1965).

${ }^{10}$ N. F. Foster, J. Appl. Phys. 34, 990 (1963).

${ }^{11}$ J. W. Tucker and V. W. Rampton, Microwave Ultrasonics in Solid-State Physics (North-Holland, Amsterdam, 1972), Chap. 3, p. 60.

${ }^{12}$ T. E. Whall, N. L. Mattey, A. D. Plews, P. J. Phillips, O. A. Mironov, R. J. Nicholas, and M. J. Kearney, Appl. Phys. Lett. 64, 357 (1994).

${ }^{13}$ D. G. Li, D. Haneman, N. S. McAlpine, and B. Chen, Phys. Rev. Lett. 73, 1170 (1994)

${ }^{14}$ A. N. Vasilev and Yu P. Gaidukov, Sov. Phys. Usp. 26, 952 (1983).

${ }^{15}$ A. N. Vasilev, V. M. Gokhfeld, and M. I. Kaganov, Sov. Phys. JETP (1968-Vol. 75, No. 6, December 1992) 65, 1286 (1987). 